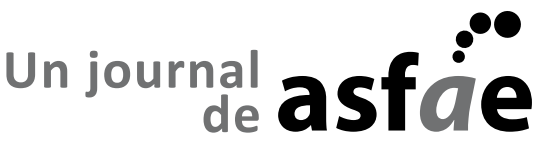

$\mathbf{m b \boldsymbol { r } ^ { 2 }}$

\section{CASO: MASISA VENEZUELA - UN MAR DE INCERTIDUMBRES}

\author{
CASE: MASISA VENEZUELA - AN UNCERTAIN OCEAN \\ Francisco Sánchez ${ }^{\mathrm{a}}$ Juan Pablo Torres ${ }^{\mathrm{b}} \bullet$ Castor Criado $^{\mathrm{c}} \cdot$ Eduardo Novoa $^{\mathrm{d}}$ \\ Clasificación: Trabajo Empírico - Estudio de Caso \\ Recibido: 23 de noviembre, 2020 / Revisado: 20 de marzo, 2021 / Aceptado: 15 de abril, 2021
}

\section{Resumen}

MASISA era un grupo empresarial chileno dedicado a la fabricación y comercialización de tableros de madera, que contaba con presencia en gran parte de América Latina y un importante volumen de exportaciones a otros continentes. Este caso se centra en las operaciones de su filial venezolana entre los años 2015 y 2018 , en los cuales la posición financiera y los resultados económicos de esta subsidiaria fueron impactados por un proceso hiperinflacionario y una importante devaluación del tipo de cambio como consecuencia de una profunda crisis económica en la que había estado inmersa Venezuela. Estos factores exógenos causaron que MASISA perdiera el control económico de sus operaciones en dicho país, lo que conllevó a que el Directorio tomara la decisión de desconsolidar las operaciones de MASISA en Venezuela a partir de octubre del 2018. Andreas Eggenberg, presidente del Directorio de MASISA, tras revisar los estados financieros del 2018 el 31 de marzo de 2019, se planteó una serie de interrogantes contables sobre los efectos que conllevaría la desconsolidación de dicha filial ¿Era la desconsolidación de la filial venezolana irreversible? y ¿cuáles serían las implicancias de la situación hiperinflacionaria en Venezuela para el futuro de MASISA?

Palabras clave: economías hiperinflacionarias, desconsolidación, cambios contables.

\begin{abstract}
MASISA was a Chilean business group dedicated to the manufacture and marketing of wooden boards and which was present in Latin America with a significant volume of exports to other continents. This case focuses on the operations of its Venezuelan subsidiary between 2015 and 2018, when the financial position and economic results of this subsidiary were affected by a hyperinflationary process and a significant devaluation of the exchange rate as a consequence of a deep economic crisis in which Venezuela had been immerse. These exogenous factors caused MASISA to lose economic control of its operations in said country, which led to the Board of Directors to make the decision to deconsolidate MASISA's operations in Venezuela in October 2018. Andreas Eggenberg, Chairman of the Board of MASISA, after reviewing the financial statements for 2018 on March 31, 2019, raised a series of accounting questions about the effects that the deconsolidation of the subsidiary would entail: Was the deconsolidation of the Venezuelan subsidiary irreversible? What would be the managerial implications of the hyperinflationary situation in Venezuela for the future of MASISA?
\end{abstract}

Keywords: Hyperinflationary economies, deconsolidation, accounting changes.

Facultad de Economía y Negocios, Universidad de Chile, Santiago, Chile. Correo electrónico: fsanchez@,fen.uchile.cl Facultad de Economía y Negocios, Universidad de Chile, Santiago, Chile. Correo electrónico: jtorresc@fen.uchile.cl Facultad de Economía y Negocios, Universidad de Chile, Santiago, Chile. Correo electrónico: ccriado@fen.uchile.cl

Facultad de Economía y Negocios, Universidad de Chile, Santiago, Chile. Correo electrónico: enovoav@fen.uchile.cl

* Los diálogos de este caso han sido redactados con fines académicos y no son opiniones expresadas en documentos públicos por las personas individualizadas. Las notas de enseñanza de este caso están disponibles a solicitud. Por favor contactar a los autores. 


\section{Introducción}

Era el último día del primer trimestre del 2019 y Andreas Eggenberg, presidente del Directorio de la compañía chilena MASISA, fiel a su costumbre, estaba sentado en su oficina pensando sobre los eventos que acontecieron durante el 2018, mientras esperaba los estados financieros de la compañía correspondientes a dicho año. Sin duda, sus pensamientos eran dominados por la incertidumbre sobre la operación de la filial venezolana de MASISA, lastrada por un proceso hiperinflacionario y una severa depreciación de la moneda local, lo que era consecuencia de la crisis económica y política en la que había estado inmersa Venezuela desde el 2013 (ver Tabla 1, Anexo I). Por ello, durante el 2018 se tomaron varias decisiones importantes, siendo una de estas la desconsolidación de los estados financieros de la filial venezolana de MASISA (MASISA, 2019b). No obstante, Andreas aún tenía dudas sobre los aspectos contables y las implicancias sobre la gestión que acarreaba la desconsolidación de una filial que operaba en una economía hiperinflacionaria. Estas interrogantes le generaban preocupación debido a que se acercaba la fecha en que iba a celebrarse la próxima Junta de Accionistas y, probablemente, sería un tema a tratar en dicha reunión.

\section{¿Qué era MASISA?}

El grupo empresarial chileno MASISA era uno de los principales productores y comercializadores de tableros de madera para muebles y arquitectura de interiores en Latinoamérica. Su origen se remonta al año 1960 como la primera empresa productora de tableros aglomerados en Chile. Su crecimiento fue paulatino a lo largo de los años con hitos importantes tales como cuando, en el año 1970, comenzó a transar sus acciones en la Bolsa de Valores de Santiago de Chile o cuando, en 1992, inició su aventura internacional desembarcando primero en el mercado argentino, para, unos años más tarde, constituir sus primeras sociedades tanto en Brasil como en Venezuela. A finales del 2018, la compañía desarrollaba su actividad en Chile, México y Venezuela, con una capacidad instalada anual de 1611000 metros cúbicos para la fabricación de tableros y 873000 metros cúbicos para el recubrimiento de tableros con papeles melamínicos y folios, además de una capacidad anual de 396000 metros cúbicos de madera aserrada y procesos de remanufactura para la fabricación de molduras de fibropanel de densidad media (ver Tabla 2, Anexo II) (MASISA, 2019b).

En cuanto a sus actividades operativas y comerciales, estas se concentraban en América Latina en donde el $70.8 \%$ de los ingresos consolidados en el 2018 fue generado. El resto, el $29.2 \%$, de los ingresos consolidados fueron resultado de las exportaciones a otros mercados, principalmente al continente asiático y América del Norte. Los ingresos de MASISA se generaban de la comercialización de dos líneas de negocio: comercial y forestal (ver Tabla 3, Anexo III). El negocio comercial se encontraba dividido en mobiliario e interiores. El primero ofrecía una variedad de diseños y texturas para el desarrollo de proyectos de fabricación de muebles de baño, cocinas, oficinas, además de tableros de fibropanel de densidad media (MDF, por sus siglas en inglés) o tableros de partículas de densidad media (MDP, por sus siglas en inglés) recubiertos por una o dos caras con películas decorativas y con protección anti-microbiana. En cambio, el área de interiores ofrece soluciones de revestimientos decorativos y acústicos, además de molduras para la terminación en los encuentros pared-piso, pared-techo y en marcos de puertas y ventanas. Por su parte, el negocio forestal se centraba en desarrollar sinergias necesarias para el abastecimiento de las plantas industriales a través de la generación de productos forestales obtenidos directamente de sus bosques, así como también a través de la recuperación de subproductos de la industria de aserrío propia y de terceros, clientes que la unidad forestal se encargaba de abastecer. Al 31 de diciembre del 2018 el patrimonio forestal de MASISA era de 298753 hectáreas de terrenos en las se incluían 188368 hectáreas de plantaciones forestales (ver Tabla 4, Anexo IV) (MASISA, 2019b).

Cabe destacar que, desde inicios del 2017, MASISA había estado inmersa en un proceso de transformación que consistía en la adaptación de su modelo de negocio, pasando de un modelo con base en una alta participación de activos industriales para la generación de valor hacia un nuevo modelo enfocado en soluciones que satisficieran las necesidades de los clientes, poniéndolos a éstos y sus necesidades en el centro de la estrategia de la compañía. Este proceso implicó la desinversión de activos industriales (Argentina, México y Brasil), la reducción de la deuda financiera (con lo recaudado con la venta de inversiones), el redimensionamiento de su estructura organizacional y un nuevo enfoque estratégico (MASISA, 2018b). En la Figura 1 del Anexo V se presenta más información de la historia de MASISA.

\section{MASISA en Venezuela}

El primer contacto de MASISA con Venezuela fue en el año 1997 con la creación de la sociedad Terranova de Venezuela C.A. que, en ese entonces, inició la construcción de un aserradero y una planta de secado, cuya puesta en marcha se dio en el 2001. Con la operación de este complejo industrial, MASISA buscaba potenciar su presencia tanto en el mercado local venezolano como en el 
mercado colombiano, ambos países sumaban un total de 70 millones de consumidores (Diario Expansión, 2001).

A finales del 2018 MASISA contaba con 147340 hectáreas forestales en Venezuela, siendo el 27\% de su propiedad y el $73 \%$ restante propiedad del Gobierno venezolano, respecto del cual la compañía tenía el 100\% de los derechos de usufructo y arriendo. El patrimonio forestal de Venezuela representaba el 49\% del total de MASISA en la región y constituía un factor clave a la hora no solo de suministrar y abastecer a las plantas industriales de la empresa, sino también de reforzar su estrategia de diversificación de riesgo al mantener sus operaciones productivas en distintos países (MASISA, 2019b).

\section{Venezuela rumbo a una crisis económica}

Desde el 2013 y a pesar de ser uno de los países con mayores recursos naturales del mundo, Venezuela había experimentado una crisis económica, social y política sin precedentes en la historia reciente de Latinoamérica. La crisis económica surgió desde que Hugo Chávez tomó el poder en el año 1999 e inició una serie de programas de elevado gasto público que generaban una alta presión sobre las arcas del Estado, las cuales dependían de los ingresos procedentes del petróleo. Así, y hasta la llegada de la crisis financiera mundial del 2008, las políticas sociales se implementaron con éxito. Pero la recesión económica $\mathrm{y}$, posteriormente con la fuerte contracción de los precios del petróleo, la situación de bonanza se convirtió en una crisis de liquidez. A pesar de las señales de alerta de reducción de gastos, Venezuela incrementó el gasto público (Pampillón y Zapata, 2019). Al cierre del año 2014, la inflación alcanzó un $57 \%$, el producto interno bruto (PIB) del país experimentó una caída de un $3.9 \%$ y el nivel de desempleo se aumentó a un $7.9 \%$ de la población activa (ver Tabla 1, Anexo I).

\section{Se agudiza la crisis económica en Venezuela en los años 2015 y 2016}

En el año 2015, el PIB de Venezuela experimentó una caída de un $6.2 \%$, debido a la disminución del precio del petróleo y a un débil contexto institucional que el país atravesaba (Salces, 2019). La inflación anual alcanzó un $180 \%$ y su moneda experimentó un deterioro respecto al dólar estadounidense (USD) de un $1544 \%$ en comparación al 2014 (ver Tabla 1, Anexo I). Como consecuencia de los vaivenes económicos que estaba viviendo dicho país y de la importante devaluación que presentaba su moneda, los estados financieros de la filial venezolana de MASISA debieron ser ajustados al tipo de cambio del Sistema Marginal de Divisas (SIMADI) para que, de esta manera, pudieran ser consolidados con la información de su empresa controladora. Este mecanismo para determinar el tipo de cambio había tomado mayor importancia para cubrir las necesidades de adquisición de dólares en el mercado venezolano, ya que permitía a personas naturales y empresas comprar y vender efectivo y títulos de valores en moneda extranjera en el mercado financiero a través de subastas periódicas cuyos criterios de asignación no se basan únicamente en el precio (MASISA, 2016b). Sobre este punto, Roberto Salas, gerente general de MASISA, manifestó: "Desde el mes de Julio en adelante, decidimos ajustar nuestros números en Venezuela al tipo de cambio SIMADI, considerando en ese momento la mejor alternativa para representar nuestros estados financieros. Este ajuste si bien tuvo impactos relevantes en patrimonio y resultados, se consideró oportuno y necesario" (MASISA, 2016b, p. 8).

Dicha filial de MASISA representaba un peso importante para el holding. Por consiguiente, el deterioro cambiario que estaba experimentando el país tuvo efectos negativos significativos en la posición financiera de la compañía a nivel consolidado. Por un lado, su patrimonio contable consolidado disminuyó en un $36.1 \%$, pasando de tener un valor de 1179 millones de USD en el 2014 a 754 millones de USD a finales del 2015 (ver Tabla 5 y Tabla 6, Anexo VI). Por otro lado, los ingresos que fueron reportados por su filial en Venezuela disminuyeron en un $67 \%$ respecto al año anterior, pasando de representar un $27 \%$ a nivel consolidado en el 2014 a solo un $13 \%$ en el 2015 (ver Tablas 7, 8, 9 y 10, Anexo VII). Asimismo, la utilidad antes de intereses, impuestos, depreciaciones y amortizaciones (Ebitda, por sus siglas en inglés) aportado desde Venezuela disminuyó en un 58\%, que alcanzó los 24 millones de USD al cierre del 2015 y representó un 15\% del Ebitda consolidado (ver Tablas 7, 8, 9 y 10, Anexo VII).

En el 2016 la crisis a nivel general de Venezuela continuaba acentuándose. En particular, el PIB venezolano se contrajo en un $16 \%$ y la inflación alcanzó un nivel del $500 \%$. Por su parte, la escasez de bienes causó que en varios períodos de dicho año se paralizaran las actividades productivas de las empresas por la falta de insumos y de repuestos (Charner, Hernández y Newton, 2016). Por ello, no era extraño que el tipo de cambio en el 2016 experimentará un deterioro de un $242 \%$ respecto al año anterior (ver Tabla 1, Anexo I). Respecto al año 2015, las ventas de dicha filial al cierre del 2016 experimentaron una baja del $4 \%$ y el Ebitda disminuyó en un 27\% (ver Tablas 7, 8, 9 y 10, Anexo VII).

A pesar de las dificultades, el negocio en Venezuela continuaba siendo importante para MASISA, lo que se evidenciaba en que el $14 \%$ del Ebitda consolidado era 
aportado por sus operaciones en Venezuela (ver Tablas 7, 8, 9 y 10, Anexo VII). Por ello, Roberto Salas monitoreaba constantemente las operaciones y las cifras financieras de su filial venezolana y cuya preocupación se reflejó en una charla de pasillo con Andreas Eggenberg:

Roberto Salas: Andreas me parece pertinente que pidamos tanto al equipo de contabilidad como de finanzas que nos envien informes cada 15 días sobre los números de Venezuela. ¿Qué te parece?

Andreas Eggenberg: Roberto estoy 100\% de acuerdo con lo que propones. La próxima semana citaré a una reunión con los equipos.

\section{La situación comienza a torcerse de manera expo- nencial}

Con la llegada de un nuevo año la incertidumbre sobre lo que podía acontecer con la filial venezolana volvió a instaurarse. A comienzos del 2017, Andreas Eggenberg y su grupo de colaboradores cercanos confiaban en una recuperación en dicho país debido a cambios que podrían ocurrir en su régimen político (DW, 2016; Pardo, 2015). En un breve diálogo Andreas Eggenberg manifestaba su entusiasmo a Roberto Salas:

Andreas Eggenberg: Roberto si finalmente ocurre un referéndum revocatorio del poder presidencial, sin duda alguna la economía venezolana se recuperará y con ella los resultados de nuestra filial.

Roberto Salas: Esperemos que esto pueda alcanzarse Andreas, sin duda, considero al igual que tú que si se consigue realizar el referéndum la situación en Venezuela puede cambiar drásticamente y con esto mejorar los números de nuestra operación en dicho país.

No obstante, este cambio en el régimen político no se dio y cualquier atisbo de cambio en el poder institucional en un futuro cercano era improbable (Ocando, 2017). Así, la esperanza de una recuperación económica desaparecía. Al cierre del 2017, el declive de la economía venezolana era profundo, lo que quedaba reflejado en: a) una inflación que alcanzó una cifra record del $2457 \%$, b) una devaluación del tipo de cambio del $397 \%$, c) un índice de desempleo que rondaba el $27.1 \%$ y d) una caída del PIB de un $14 \%$ (ver Tabla 1, Anexo I).

Respecto a la filial venezolana de MASISA, los ingresos se incrementaron en el 2017 en 264 millones de USD, lo que representaba un incremento del 103\% respecto al 2016 (ver Tablas 7, 8, 9 y10, Anexo VII). Sin embargo, los volúmenes de sus ingresos domésticos disminuyeron un $47 \%$ y no pudieron ser compensados por las exportaciones desde dicho país (Feller Rate, 2018). Esta relación inversa entre volúmenes y valor de venta tuvo lugar principalmente debido a un alza en la inflación en 2017 que fue compensada parcialmente por una devaluación del bolívar respecto al dólar americano (ver Tabla 1, Anexo I). A pesar de contar con una mayor cifra de ingresos en el 2017, el Ebitda de la empresa venezolana se desplomó en un $64 \%$ en comparación al año anterior, situándose aproximadamente en los 6 millones de USD para finales del 2017, monto que representaba solamente el 5\% del Ebitda consolidado de MASISA (ver Tablas 7, 8, 9 y 10, Anexo VII).

\section{Un año 2018 de cambios contables}

A inicios del 2018, el directorio de MASISA tomó la decisión de modificar la moneda funcional de su filial venezolana, pasó del bolívar fuerte al dólar estadounidense. Esto se justificaba debido a que la mayor parte de los ingresos de la filial venezolana habían sido obtenidos en dólares estadounidenses, como consecuencia de que las exportaciones al cierre del 2017 superaron el 50\% del total de la producción local. Se esperaba que esta tendencia fuese en aumento en los siguientes años, razón por la cual el mercado de exportación había tomado un rol relevante en la obtención de los flujos de caja necesarios para la continuidad de las operaciones de la filial venezolana. Del mismo modo, a partir del 2018, con el objetivo de reflejar más fielmente el valor de las transacciones económicas que se realizaron en moneda local, la compañía comenzó a utilizar como referencia el tipo de cambio en el que se liquidaban las exportaciones en vez del tipo de cambio SIMADI (MASISA, 2019b).

Por su parte, la economía venezolana durante el 2018 empeoraba continuamente, las políticas macroeconómicas que se llevaron a cabo en dicho país no lograban contener el proceso hiperinflacionario ni la fuerte devaluación de la moneda, lo que intensificó aún más la crisis en la que estaba inmersa Venezuela (Laya y Rosati, 2018). Los principales indicadores económicos del año 2018 eran el reflejo fiel de la coyuntura que se vivía en dicho país. En particular, el desempleo alcanzó una tasa del 35\%, la inflación era abismal (1370000\%) y el PIB experimentó la caída más importante desde el 2014 (-18\%) (ver Tabla 1, Anexo I). Este deterioro exponencial en la economía de Venezuela dificultaba medir con fiabilidad los resultados de la operación de MASISA en dicho país. Por ello, la administración de MASISA decidió, según lo que establece el párrafo $\mathrm{N}^{\circ} 8$ de la Norma Internacional de Información Financiera $\mathrm{N}^{\mathrm{o}} 10$ (Estados financieros consolidados), desconsolidar las operaciones 
de su filial en Venezuela desde el 1 de octubre de 2018 (ver Tabla 11 y Tabla 12, Anexo VIII) (MASISA, 2019b).

Esta decisión tuvo impacto en la posición financiera a nivel consolidado, ya que implicó realizar ajustes como si la compañía estuviese enfrentando la enajenación de la filial venezolana. En específico, MASISA reconoció en sus estados financieros consolidados una pérdida de 284.5 millones de USD en resultados producto de la desconsolidación (ver Tablas 7, 8, 9 y 10, Anexo VII). Este ajuste se llevó a cabo debido a que MASISA tuvo que absorber los ajustes por tipo de cambio históricos que habían sido reconocidos en el patrimonio relacionados a su inversión en la sociedad venezolana. Adicionalmente, como consecuencia de la desconsolidación, MASISA reconoció en una línea de sus estados financieros consolidados la inversión a valor justo de la filial venezolana en la cuenta otros activos financieros no corrientes, el cual correspondió a 31.6 millones de USD. Todos estos ajustes generados por la desconsolidación de la filial venezolana dieron como resultado un efecto positivo en el patrimonio consolidado de MASISA por 20.5 millones de USD (MASISA, 2019a, 2019b).

Tras llevar a cabo este cambio contable las cifras consolidadas de MASISA al cierre del 2018 consideraba solamente 9 meses de operación de la filial venezolana y mostraban un Ebitda consolidado de 86.2 millones de USD, de los cuales solo el 1\% correspondía al aporte del país venezolano (ver Tablas 7, 8, 9 y 10, Anexo VII). Tras este cambio contable, el aporte a las ventas y el Ebitda de la filial venezolana al Grupo MASISA quedaban atrás.

\section{Filial venezolana, ¿un cambio contable necesario?}

Andreas Eggenberg sabía que el efecto contable de la desconsolidación de la filial venezolana de MASISA podría ser un tema para discutir en la próxima Junta General de Accionistas que iba a celebrarse el 24 de abril del 2019. Tras recibir y analizar los estados financieros consolidados del 2018, se trasladó a la oficina del gerente general corporativo, Roberto Salas, y mantuvieron un breve diálogo':

Andreas Eggenberg: Roberto, ¿entiendes los cambios que causó la desconsolidación de la filial venezolana sobre las cifras que muestran los estados financieros?

Roberto Salas: El otro día me trataron de explicar, pero lo único que me quedo claro que fue necesario por la situación de extrema incertidumbre e imposibilidad

Los diálogos de este caso han sido redactados con fines académicos y no son opiniones expresadas en documentos públicos por las personas individualizadas. de manejar la inconsistencia de las variables macroeconómicas.

Tras este diálogo, Andreas Eggenberg citó a una reunión urgente al personal contable de la compañía para buscar respuestas a las dudas de índole contable, a saber: ¿qué cambios causó la desconsolidación en las cifras de los estados financieros consolidados de MASISA? y ¿cuál sería el impacto de este cambio contable sobre los principales indicadores financieros del Grupo MASISA? Aunque, sus mayores interrogantes fueron sobre si era irreversible la desconsolidación de la filial venezolana y ¿cuáles serían las implicancias de la situación hiperinflacionaria en Venezuela para el futuro de MASISA?

\section{Referencias}

Charner, F., Hernández, O., \& Newton, P. (1 de junio de 2016). La escasez de productos básicos aumenta en Venezuela. Diario Expansión. https://expansion.mx/ economia/2016/06/01/la-escasez-de-productos-basicos-aumenta-en-venezuela

Diario Expansión. (2001). Población de Colombia 2001. https://datosmacro.expansion.com/demografia/poblacion/colombia? anio $=2001$

DW. (5 de enero de 2016). Venezuela: Se instaló el Parlamento con mayoría opositora. Deutsche Welle $(\mathrm{DW})$. https://www.dw.com/es/venezuela-se-instaló-elparlamento-con-mayor\%C3\%ADa-opositora/a18959735

Feller Rate. (2018). Informe de clasificación abril 2018 MASISA S.A. https://www.feller-rate.cl/general2/corporaciones/masisa1804.pdf

IndexMundi. (2018). Cuadro de datos históricos anuales. IndexMundi. https://www.indexmundi.com/g/g.aspx$? \mathrm{c}=\mathrm{ve} \& \mathrm{v}=74 \& \mathrm{l}=\mathrm{es}$

Inversiones Internacionales Terranova. (2016). Estados financieros consolidados año 2015 Inversiones Internacionales Terranova S. A.http://www.cmfchile. $\mathrm{cl} /$ institucional/mercados/ver_filial.php?archivo=fil_96802690_201512_20160307222511.pdf

Inversiones Internacionales Terranova. (2018). Estados financieros consolidados año 2017 Inversiones Internacionales Terranova $S$. A. http://www.cmfchile. $\mathrm{cl} /$ institucional/mercados/ver_filial.php?archivo=fil_96802690_201712_20180327174639.pdf

Laya, P., \& Rosati, A. (9 de octubre de 2018). Venezuela tendrá una inflación en 2018 de $1.370 .000 \%$. Diario El Financiero. https://www.elfinanciero.com.mx/ economia/venezuela-tendra-una-inflacion-de-1-37millones-por-ciento-a-finales-de-ano-estima-el-fmi 
MASISA. (2015a). Press release cuarto trimestre 2014 MASISA S.A. https://corporativo.masisa.com/inversionistas/resultados-adminstrativos/

MASISA. (2015b). Memoria anual 2014 MASISA S. A. https://corporativo.masisa.com/inversionistas/memoria-y-eeff/

MASISA. (2016a). Press release cuarto trimestre 2015 MASISA S. A. https://corporativo.masisa.com/inversionistas/resultados-adminstrativos/

MASISA. (2016b). Memoria Anual 2015 MASISA S. A. https://corporativo.masisa.com/inversionistas/memoria-y-eeff/

MASISA. (2017a). Press release cuarto trimestre 2016 MASISA S. A.https://corporativo.masisa.com/inversionistas/resultados-adminstrativos/

MASISA. (2017b). Memoria anual 2016 MASISA S. A. https://corporativo.masisa.com/inversionistas/memoria-y-eeff/

MASISA. (2018a). Press release cuarto trimestre 2017 MASISA S. A. https://corporativo.masisa.com/inversionistas/resultados-adminstrativos/

MASISA. (2018b). Memoria anual 2017 MASISA S. A. https:/corporativo.masisa.com/inversionistas/memoria-y-eeff/

MASISA. (2019a). Press release cuarto trimestre 2018 MASISA S. A. https://corporativo.masisa.com/inversionistas/resultados-adminstrativos/
MASISA. (2019b). Memoria anual 2018 MASISA S.A. https:/corporativo.masisa.com/inversionistas/memoria-y-eeff/

Ocando, G. (31 de marzo de 2017). Qué significa y qué consecuencias puede tener que el Tribunal Supremo de Venezuela asuma las funciones legislativas de la Asamblea mientras esta se mantenga en "desacato". BBC News. https://www.bbc.com/mundo/noticias-america-latina-39448001

Pampillón, R., \& Zapata, A. (27 de enero de 2019). Venezuela: El colapso total de una economía. Diario Expansión. https://www.expansion.com/latinoamerica/2019/01/27/5c4e23f1468aeb2c558b4581.html

Pardo, D. (5 de diciembre de 2015). Qué cambios puede impulsar la oposición de Venezuela con su victoria parlamentaria. BBC News. https://www.bbc.com/ mundo/noticias/2015/12/151207_analisis_venezuela_oposicion_elecciones_parlamentarias_amv

Salces, I. (26 de enero de 2019). Venezuela ha destruido la mitad de su economía en seis años. Diario Cinco Días. https://cincodias.elpais.com/cincodias/2019/01/25/economia/1548423787_186188.html

Toro, M. (3 de mayo de 2019). Estas son las impresionantes cifras de la crisis en Venezuela. CNN Español. https:// cnnespanol.cnn.com/2019/05/03/estas-son-las-impresionantes-cifras-de-la-crisis-en-venezuela/ 


\section{Anexos}

\section{Anexo I. Indicadores macroeconómicos de Venezuela}

Tabla 1. Indicadores macroeconómicos

\begin{tabular}{|c|c|c|c|c|c|}
\hline Indicador & 2014 & 2015 & 2016 & 2017 & 2018 * \\
\hline Tipo de cambio Bolívar Fuerte (VEF) x 1 USD $^{1}$ & 12 & 199 & 674 & 3345 & 6217000 \\
\hline Tipo de cambio Bolívar liquidación exportación x 1 USD $^{1}$ & & & & & 491 \\
\hline Evolución PIB ${ }^{2}$ & $-3.9 \%$ & $-6.2 \%$ & $-16.5 \%$ & $-14.0 \%$ & $-18.0 \%$ \\
\hline Desempleo $^{3}$ & $7.9 \%$ & $8.0 \%$ & $10.5 \%$ & $27.1 \%$ & $35.0 \%$ \\
\hline
\end{tabular}

* El 20 de agosto del 2018 entra en vigencia un nuevo sistema cambiario que cambió la nominación de la moneda de Bolívar Fuerte a Bolívar Soberano, lo que implicó que, a partir de esa fecha, 1 Bolívar Soberano equivale a 100.000 Bolívares Fuertes.

Fuente: ${ }^{1}$ MASISA (2015b, 2016b, 2017b, 2018b, 2019b). ${ }^{2}$ Pampillón y Zapata (2019). ${ }^{3}$ IndexMundi (2018) y Toro (2019).

\section{Anexo II. Capacidad de producción de MASISA}

Tabla 2. Capacidad de producción por país al cierre del 2018 (en miles de metros cúbicos)

\begin{tabular}{|c|c|c|c|c|c|c|c|}
\hline País & Planta & $\mathbf{P B}^{1}$ & MDF & MDP & Melamina & Aserradero & Molduras MDF \\
\hline \multirow{2}{*}{ Chile } & Mapal & 92 & 150 & & 260 & & \\
\hline & Cabrero & & 350 & 280 & 228 & 212 & 184 \\
\hline \multirow[t]{2}{*}{ México } & Chihuahua & 180 & & & 76 & & \\
\hline & Zitacuaro & 184 & & & 103 & & \\
\hline \multicolumn{2}{|c|}{ Total sin Venezuela } & 611 & 720 & 280 & 873 & 212 & 184 \\
\hline
\end{tabular}

${ }^{1}$ Tableros de partículas (PB, por sus siglas en inglés), también conocido como aglomerado.

Fuente: MASISA (2019b).

\section{Anexo III. Variedad de productos de MASISA}

\section{Tabla 3. Productos comercializados}

\begin{tabular}{ll}
\hline \multicolumn{1}{c}{ Producto } & Descripción \\
Tableros melamínicos & Tableros de PB, MDP o MDF. \\
Molduras MDF & $\begin{array}{l}\text { Utilizados para realizar terminaciones en encuentros pared-piso, } \\
\text { pared-techo y en marcos de puertas y ventanas. } \\
\text { Tapacantos }\end{array}$ \\
y darle un acabado perfecto a muebles y proyectos.
\end{tabular}




\begin{tabular}{|c|c|c|}
\hline Producto & Descripción & Imagen \\
\hline Revestimientos interiores & $\begin{array}{l}\text { Soluciones decorativas modulares para revestir paredes y espacios } \\
\text { interiores de forma rápida, simple y limpia. }\end{array}$ & \\
\hline Tableros MDP & $\begin{array}{l}\text { Tableros de partículas de madera especialmente indicados para la } \\
\text { producción de muebles de líneas rectas o formas orgánicas. }\end{array}$ & \\
\hline Tableros PB & $\begin{array}{l}\text { Tableros de partículas de madera que se caracterizan por ser resistentes } \\
\text { y livianos. }\end{array}$ & \\
\hline Madera aserrada & $\begin{array}{l}\text { Se utilizan principalmente en la fabricación de embalajes o pallets, } \\
\text { muebles y en construcción. }\end{array}$ & \\
\hline
\end{tabular}

Fuente: MASISA (2019b).

Anexo IV. Plantaciones forestales de MASISA S.A.

Tabla 4. Hectáreas de plantaciones forestales por país al cierre del 2018

\begin{tabular}{|c|c|c|c|c|}
\hline Tipo de plantación & Chile & Argentina & Venezuela & Total \\
\hline Plantaciones pino & 48026 & 23681 & 87391 & 159098 \\
\hline Plantaciones eucaliptus & 303 & 21385 & 16 & 21704 \\
\hline Total plantaciones forestales & 48469 & 45066 & 94833 & 188368 \\
\hline Terrenos por forestar & 7720 & 9332 & 19974 & 37026 \\
\hline Total terrenos & 79030 & 72383 & 147340 & 298753 \\
\hline
\end{tabular}

Fuente: MASISA (2019b). 
Figura 1. Breve reseña histórica 1

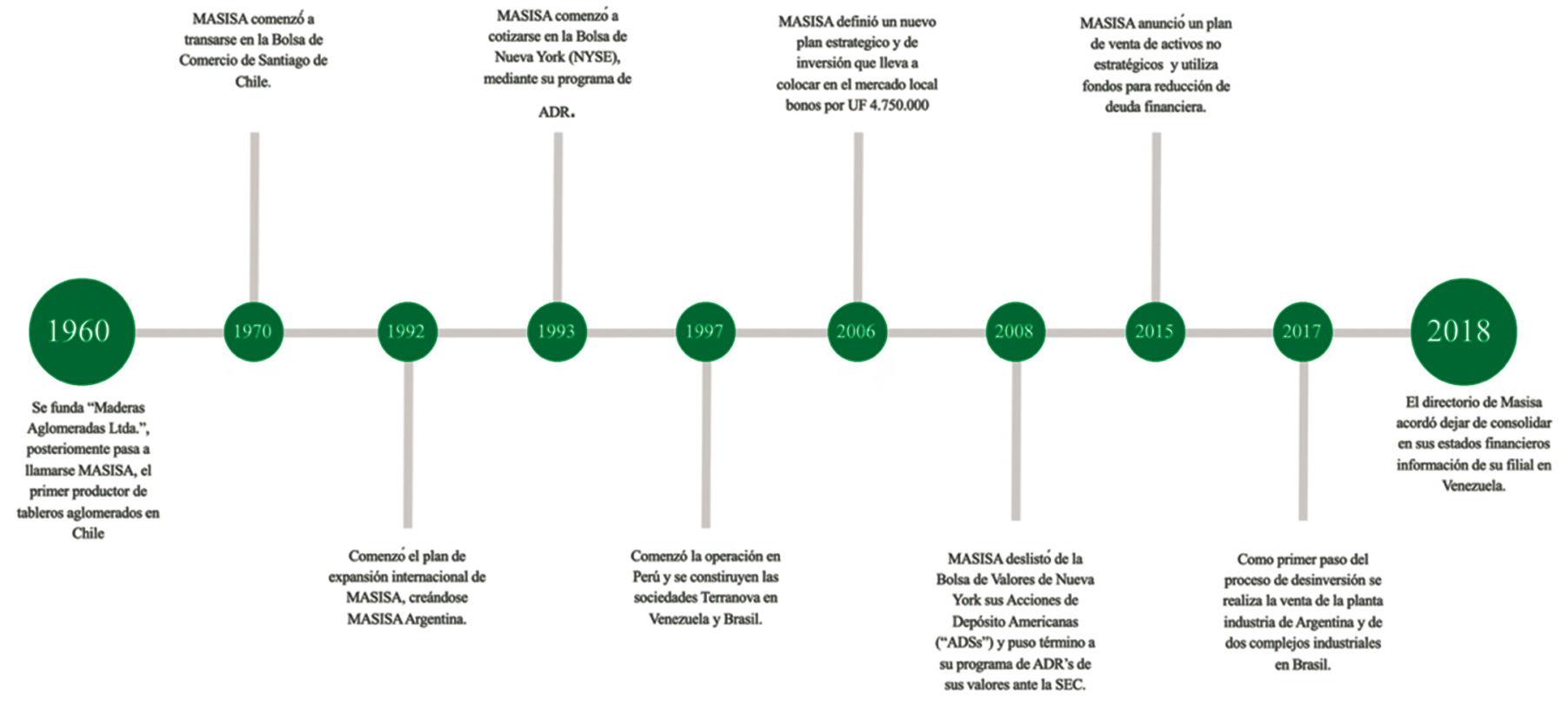

${ }^{1} 1000000$ Unidades de Fomento (UF) eran equivalentes a 186.9 millones de USD al 31 de diciembre del 2016.

Fuente: MASISA (2019b).

Anexo VI. Cifras del estado de situación financiera consolidado de MASISA

Tabla 5. Estado de situación financiera consolidado resumido

\begin{tabular}{|c|c|c|c|c|c|}
\hline Cifras en miles de USD & 2014 & 2015 & 2016 & 2017 & 2018 \\
\hline Activo corriente total & 721390 & 516452 & 457407 & 618527 & 531730 \\
\hline Activo no corriente total & 1704046 & 1394311 & 1332724 & 998376 & 954503 \\
\hline Pasivo corriente total & 325676 & 317464 & 334385 & 341691 & 270817 \\
\hline Pasivo no corriente total & 920476 & 839308 & 698645 & 494200 & 438946 \\
\hline Pasivo total & 1246152 & 1156772 & 1033030 & 835891 & 709763 \\
\hline Pasivo y patrimonio total & 2425436 & 1910763 & 1790131 & 1616903 & 1486233 \\
\hline
\end{tabular}

Fuente: MASISA (2015b, 2016b, 2017b, 2018b, 2019b).

Tabla 6. Otras reservas (ítem de patrimonio)

\begin{tabular}{lcc}
\hline \multicolumn{1}{c}{ Cifras en miles de USD } & $\mathbf{2 0 1 7}$ & $\mathbf{2 0 1 8}$ \\
\hline Para futuros dividendos & 51424 & 51424 \\
De conversión & -566958 & -223629 \\
Revaluaciones de terrenos forestales & 20999 & 114800 \\
Ganancias y pérdidas por planes de beneficios definidos & -3269 & -3251 \\
De coberturas & 10216 & 1572 \\
Otras & -278 & -278 \\
\hline
\end{tabular}

Fuente: MASISA (2019b). 
Anexo VII. Cifras del estado de resultados consolidado de MASISA

Tabla 7. Estado de resultados

\begin{tabular}{lcccccccccc}
\hline \multicolumn{1}{c}{ Cifras en miles de USD } & $\mathbf{2 0 1 4}$ & $\mathbf{\%}$ & $\mathbf{2 0 1 5}$ & $\mathbf{\%}$ & $\mathbf{2 0 1 6}$ & $\mathbf{\%}$ & $\mathbf{2 0 1 7}$ & $\mathbf{\%}$ & $\mathbf{2 0 1 8}$ & $\mathbf{\%}$ \\
\hline Ingresos & 1544623 & $100 \%$ & 1052555 & $100 \%$ & 959841 & $100 \%$ & 1057414 & $100 \%$ & 612408 & $100 \%$ \\
Costo de venta & -1243746 & $-81 \%$ & -825536 & $-78 \%$ & -760795 & $-79 \%$ & -850492 & $-80 \%$ & -512489 & $-84 \%$ \\
Ganancia bruta & $\mathbf{3 0 0 8 7 7}$ & $\mathbf{1 9 \%}$ & $\mathbf{2 2 7 0 1 9}$ & $\mathbf{2 2 \%}$ & $\mathbf{1 9 9 0 4 6}$ & $\mathbf{2 1 \%}$ & $\mathbf{2 0 6 9 2 2}$ & $\mathbf{2 0 \%}$ & $\mathbf{9 9 9 2 9}$ & $\mathbf{1 6 \%}$ \\
Gastos de adm. y distribución & -213542 & $-14 \%$ & -145185 & $-14 \%$ & -145364 & $-15 \%$ & -163477 & $-15 \%$ & -78754 & $-13 \%$ \\
Otros gastos e ingresos ${ }^{1}$ & 40820 & $3 \%$ & 78480 & $7 \%$ & 47085 & $5 \%$ & -389424 & $-37 \%$ & -344843 & $-56 \%$ \\
Costos financieros netos & -55613 & $-4 \%$ & -49844 & $-5 \%$ & -46653 & $-5 \%$ & -62976 & $-6 \%$ & -32876 & $-5 \%$ \\
Diferencias de cambio & -21740 & $-1 \%$ & -40920 & $-4 \%$ & -12815 & $-1 \%$ & 6869 & $1 \%$ & 5265 & $1 \%$ \\
Reajustes & -44313 & $-3 \%$ & -15724 & $-1 \%$ & -20204 & $-2 \%$ & 15556 & $1 \%$ & 442 & $0 \%$ \\
Impuestos & -6667 & $0 \%$ & -8216 & $-1 \%$ & -21246 & $-2 \%$ & 27876 & $3 \%$ & 125469 & $20 \%$ \\
Ganancia (pérdida) neta & $\mathbf{- 1 7 8}$ & $\mathbf{0 \%}$ & $\mathbf{4 5 6 1 0}$ & $\mathbf{4 \%}$ & $\mathbf{- 1 5 1}$ & $\mathbf{0 \%}$ & $\mathbf{- 3 5 8 6 5 4}$ & $\mathbf{- 3 4 \%}$ & $\mathbf{- 2 2 5 3 7 1}$ & $\mathbf{- 3 7 \%}$ \\
\hline
\end{tabular}

${ }^{1}$ El 2017 y 2018 incluye las pérdidas reconocidas por única vez (ver tabla 10).

Fuente: MASISA (2015b, 2016b, 2017b, 2018b, 2019b).

Tabla 8. Ingresos por país

\begin{tabular}{lccccc}
\hline \multicolumn{1}{c}{ Cifras en miles de USD } & $\mathbf{2 0 1 4}$ & $\mathbf{2 0 1 5}$ & $\mathbf{2 0 1 6}$ & $\mathbf{2 0 1 7}$ & $\mathbf{2 0 1 8}$ \\
\hline Chile & 593135 & 441021 & 382977 & 350004 & 133234 \\
Argentina & 177632 & 198933 & 153575 & 1872 \\
México & 151373 & 131569 & 142056 & 163899 \\
Brasil & 213158 & 145253 & 150695 & 145923 & 264354 \\
Venezuela & 409325 & 135780 & 130538 & $\mathbf{9 5 9 8 4 1}$ & $\mathbf{1 0 5 7 4 1 4}$ \\
Ingreso total & $\mathbf{1 5 4 4 6 2 3}$ & $\mathbf{1 0 5 2 5 5 5}$ & $\mathbf{6 1 2 4 1 8}$ \\
\hline
\end{tabular}

Fuente: MASISA (2015a, 2016a, 2017a, 2018a, 2019a).

Tabla 9. Ebitda recurrente por país ${ }^{1}$

\begin{tabular}{|c|c|c|c|c|c|c|c|c|c|c|}
\hline Cifras en miles de USD & 2014 & $\%$ & 2015 & $\%$ & 2016 & $\%$ & 2017 & $\%$ & 2018 & $\%$ \\
\hline Chile + Región Andina & 44050 & $23 \%$ & 42800 & $27 \%$ & 50100 & $40 \%$ & 54800 & $42 \%$ & 51500 & $60 \%$ \\
\hline Argentina & 50260 & $26 \%$ & 56800 & $36 \%$ & 40400 & $32 \%$ & 34900 & $27 \%$ & 12800 & $15 \%$ \\
\hline México & 12300 & $6 \%$ & 17100 & $11 \%$ & 21100 & $17 \%$ & 25200 & $19 \%$ & 20900 & $24 \%$ \\
\hline Venezuela & 56190 & $29 \%$ & 23600 & $15 \%$ & 17200 & $14 \%$ & 6100 & $5 \%$ & 1000 & $1 \%$ \\
\hline Gastos Corporativos & & $0 \%$ & & & -9200 & $-7 \%$ & -6600 & $-5 \%$ & & $0 \%$ \\
\hline Ebitda total & 190500 & $100 \%$ & 156100 & $100 \%$ & 126300 & $100 \%$ & 131400 & $100 \%$ & 86200 & $100 \%$ \\
\hline
\end{tabular}

${ }^{1}$ Cifras de operaciones comparables.

Fuente: MASISA (2015a, 2016a, 2017a, 2018a, 2019a).

Tabla 10. Efectos de pérdidas reconocidas por única vez en MASISA

\begin{tabular}{lcc}
\hline \multicolumn{1}{c}{ Cifras en miles de USD } & $\mathbf{2 0 1 7}$ & $\mathbf{2 0 1 8}$ \\
\hline Venezuela & -153600 & -284500 \\
Plan desinversión Argentina-Brasil & -102300 & 33700 \\
Reestructuración / Reorganización & -8500 & -4000 \\
Gastos reestructuración financiera & -8200 & -28900 \\
México & & 5600 \\
Otros impactos & & $\mathbf{- 2 7 8 1 0 0}$ \\
Pérdidas reconocidas por única vez & $\mathbf{- 2 7 2 ~ 6 0 0}$ & $\mathbf{2 0 0}$ \\
\hline
\end{tabular}

Fuente: MASISA (2018a, 2019a). 
Anexo VIII. Cifras de Inversiones Internacionales Terranova (filial venezolana)

Tabla 11. Estado de situación financiera consolidado resumido

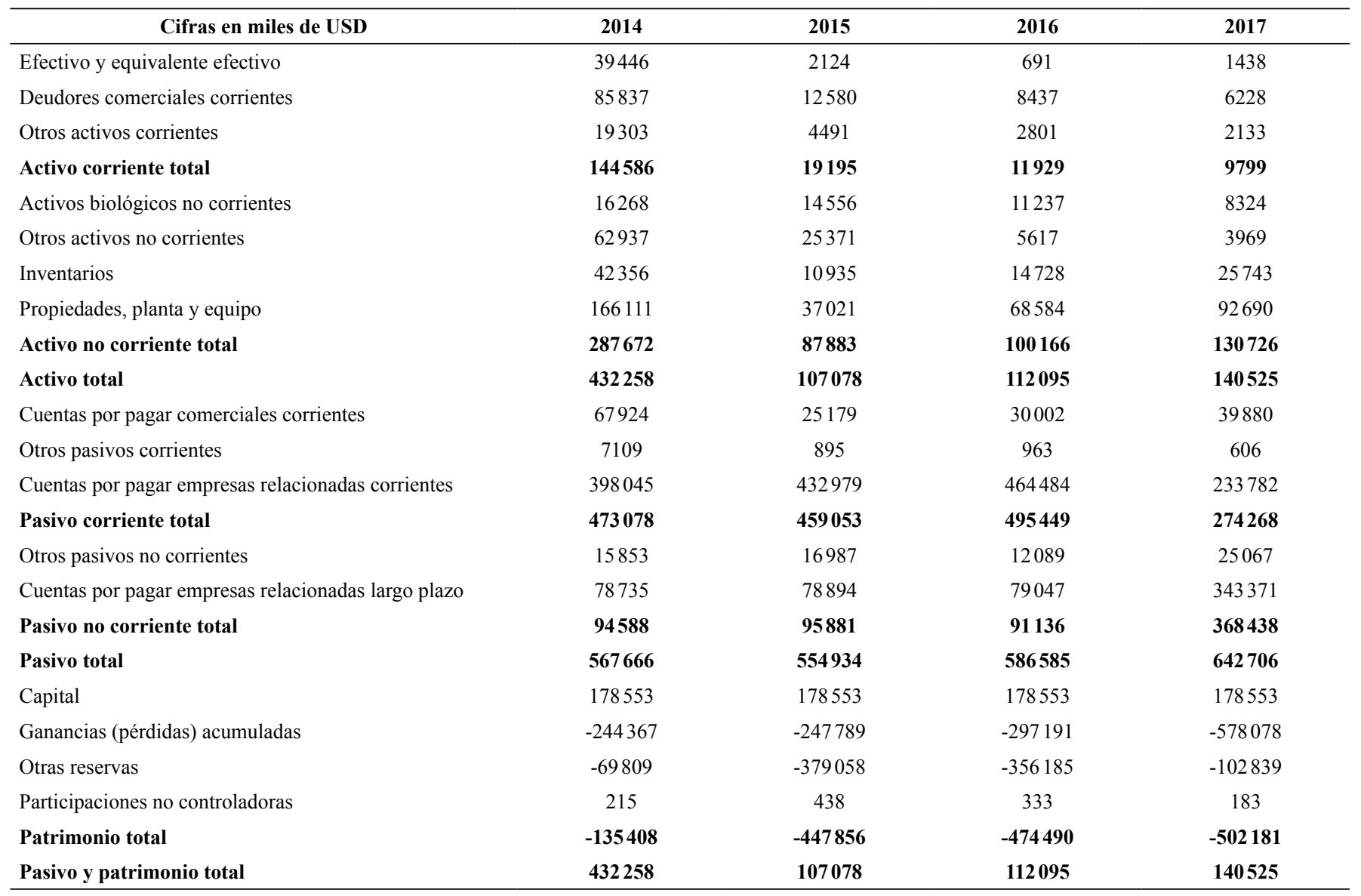

Fuente: Inversiones Internacionales Terranova $(2016,2018)$.

Tabla 12. Estado de resultados consolidado

\begin{tabular}{|c|c|c|c|c|c|c|c|c|}
\hline Cifras en miles de USD & 2014 & $\%$ & 2015 & $\%$ & 2016 & $\%$ & 2017 & $\%$ \\
\hline Ingresos & 418408 & $100 \%$ & 130052 & $100 \%$ & 130462 & $100 \%$ & 264509 & $100 \%$ \\
\hline Costo de venta & -300560 & $-72 \%$ & -88004 & $-68 \%$ & -92753 & $-71 \%$ & -200443 & $-76 \%$ \\
\hline Ganancia bruta & 117848 & $28 \%$ & 42048 & $32 \%$ & 37709 & $29 \%$ & 64066 & $24 \%$ \\
\hline Gastos de adm. y distribución & -64781 & $-15 \%$ & -20210 & $-16 \%$ & -25939 & $-20 \%$ & -58248 & $-22 \%$ \\
\hline Otros gastos e ingresos & -8278 & $-2 \%$ & 10314 & $8 \%$ & 1749 & $1 \%$ & -260002 & $-98 \%$ \\
\hline Costos financieros netos & -26655 & $-6 \%$ & -32925 & $-25 \%$ & -34277 & $-26 \%$ & -33424 & $-13 \%$ \\
\hline Diferencias de cambio & -8974 & $-2 \%$ & -11067 & $-9 \%$ & 342 & $0 \%$ & 23740 & $9 \%$ \\
\hline Reajustes & -44321 & $-11 \%$ & -15683 & $-12 \%$ & -20176 & $-15 \%$ & 3860 & $1 \%$ \\
\hline Impuestos & 6623 & $2 \%$ & 10214 & $8 \%$ & -9117 & $-7 \%$ & -22268 & $-8 \%$ \\
\hline Ganancia (pérdida) neta & -28538 & $-7 \%$ & -17309 & $-13 \%$ & -49709 & $-38 \%$ & -282276 & $-107 \%$ \\
\hline
\end{tabular}

Fuente: Inversiones Internacionales Terranova (2016, 2018). 